(2) OPEN ACCESS

\title{
Improved renal allograft survival for pre-emptive paediatric renal transplant recipients in the UK
}

\author{
Matko Marlais (1) , ${ }^{1}$ Kate Martin, ${ }^{2}$ Stephen D Marks (ㄱ) 1,3
}

${ }^{1}$ Department of Paediatric Nephrology, Great Ormond Street Hospital for Children NHS Foundation Trust, London, UK ${ }^{2}$ Statistics, NHS Blood and Transplant, Bristol, UK ${ }^{3}$ NIHR Great Ormond Street Hospital Biomedical Research Centre, University College London, Great Ormond Street Institute of Child Health, London, UK

\section{Correspondence to}

Dr Stephen D Marks, Renal Unit, Great Ormond Street Hospital for Children NHS Foundation Trust, London WC1N 3JH, UK; Stephen.Marks@gosh.nhs.uk

This study was previously presented as an abstract at the British Transplantation Society Congress 2018, the American Transplant Congress 2018, the Transplantation Society Congress 2018 and the International Paediatric Transplant Association Congress 2019.

Received 27 November 2020 Revised 15 February 2021 Accepted 8 March 2021 Published Online First 20 May 2021

\section{ABSTRACT}

Background The aim of this study was to investigate whether being on dialysis at the time of renal transplantation affected renal allograft survival in paediatric renal transplant recipients (pRTRs).

Methods Retrospective study of UK Transplant Registry (National Health Service Blood and Transplant) data on all children (aged $<18$ years) receiving a kidney-only transplant from 1 January 2000 to 31 December 2015. Kaplan-Meier estimates of patient and renal allograft survival calculated and Cox regression modelling accounting for donor type. The relationship between time on dialysis and renal allograft survival was examined. Results 2038 pRTRs were analysed: 607 (30\%) were pre-emptively transplanted, 789 (39\%) and 642 (32\%) on peritoneal dialysis and haemodialysis, respectively, at the time of transplantation. Five-year renal allograft survival was significantly better in the pre-emptively transplanted group (90.6\%) compared with those on peritoneal dialysis and haemodialysis $(86.4 \%$ and $85.7 \%$, respectively; $p=0.02$ ). After accounting for donor type, there was a significantly lower hazard of 5-year renal allograft failure in pre-emptively transplanted children (HR 0.742, $p=0.05$ ). Time spent on dialysis pre-transplant negatively correlated with renal allograft survival $(p=0.002)$. There was no significant difference in 5-year renal allograft survival between children who were on dialysis for less than 6 months and children transplanted pre-emptively ( $87.5 \%$ vs $90.5 \%, p=0.25$ ). Conclusions Pre-emptively transplanted children have improved 5-year renal allograft survival, compared with children on dialysis at the time of transplantation. Although increased time spent on dialysis correlated with poorer renal allograft survival, there was no evidence that short periods of dialysis pre-transplant affected renal allograft survival.

\section{INTRODUCTION}

The gold standard treatment for children with end stage kidney disease (ESKD) is pre-emptive livingdonor renal transplant, which is associated with improved outcomes with reduced mortality and morbidity. ${ }^{12}$ While there is a large body of evidence supporting the benefits of living-donor compared with deceased-donor transplantation, ${ }^{34}$ there is less evidence to support the importance of pre-emptive renal transplantation (before requiring dialysis).

A number of adult studies have shown the benefits of pre-emptive transplantation above haemodialysis (HD), but all past studies have been observational with a number of limitations. ${ }^{1}$ The evidence comparing dialysis modality pre-transplant in adults is conflicting, with some studies finding that those

\section{What is already known on this topic?}

- Living-related kidney transplantation is associated with improved outcomes in children, compared with deceased-donor kidney transplantation.

- The improved outcomes with pre-emptive kidney transplantation (before needing dialysis) are based mainly on adult evidence.

\section{What this study adds?}

- Children who receive a pre-emptive kidney transplant in the UK have improved renal allograft survival, compared with those on dialysis at the time of transplant.

- Short periods of time on dialysis ( $<6$ months) pre-transplant do not appear to significantly worsen renal allograft survival in UK paediatric renal transplant recipients.

on peritoneal dialysis (PD) have superior renal allograft survival, but others finding no differences in survival based on dialysis modality. ${ }^{5-7}$ Recent data from the French Transplant Database show a lower risk of renal allograft failure in pre-emptive transplantation, compared with kidney transplantation performed after starting dialysis, even if dialysis was for less than 6 months. ${ }^{8}$ While the adult studies are informative, there are important differences between adults and children with ESKD.

Clinicians try to avoid dialysis wherever possible, in view of the significantly associated mortality and morbidity. ${ }^{9}$ Therefore, it seems logical that avoiding dialysis prior to needing a renal transplant is likely to result in improved patient outcomes, but the impact on renal allograft survival is less clear.

While some children with ESKD have enough residual renal function that allows them to avoid dialysis while they are being prepared for a renal transplant, not all children are in this position. Some children will present in ESKD and require dialysis almost immediately, often for a short period of time while transplant preparations take place. Some children will require native nephrectomies as part of their management prior to transplantation, which will necessitate commencement of dialysis. What is also not known is whether there is any negative effect of a short period on dialysis prior to renal transplantation. This information would be important, as to delay dialysis inappropriately may cause more harm than good in certain 
situations. There is evidence to suggest that adults on dialysis for shorter periods of time pre-transplantation have improved renal allograft outcomes, compared with those on dialysis for longer periods of time. ${ }^{10}$

The primary aim of this study was to assess whether being on dialysis at the time of renal transplantation affects renal allograft survival in paediatric renal transplant recipients. Secondary aims of this study were to assess whether increasing time on dialysis has any cumulative effect on renal allograft survival, and to assess whether short periods of time on dialysis lead to poorer renal allograft survival.

\section{METHODS}

\section{Patient characteristics}

This was a retrospective study using data from the UK Transplant Registry held by National Health Service (NHS) Blood and Transplant. Data were obtained for all kidney-only transplants for paediatric recipients ( $<18$ years) from 1 January 2000 to 31 December 2015. Multiorgan and en bloc transplants were excluded from the analysis.

For the primary analysis in this study, the cohort was split into those not on dialysis at the time of transplantation (taken as the pre-emptive group), those on HD at the time of transplant and those on PD at the time of transplant. For the secondary analyses in this study, the HD and PD patients were grouped together as a single dialysis group to increase power.

\section{Statistical analysis}

Kaplan-Meier estimates were used to estimate univariate posttransplant patient and renal allograft survival across those transplanted pre-emptively, those on HD and those on PD at the time of transplant. One-year and 5-year estimates were calculated. The univariate log-rank test was used to detect a statistically significant difference in survival across the three groups. Deathcensored renal allograft survival was defined as time from renal transplantation to renal allograft failure.

A Cox proportional hazards regression model was used to explore differences in renal allograft survival, having accounted for donor type as a covariate. Donor type was tested as a potential explanatory variable and was found to be significant at the $5 \%$ level, so it was included as a covariate, as was recipient age.

To determine whether a short period of time on dialysis (arbitrarily defined as less than 6 months) affected renal allograft survival, we used Kaplan-Meier estimates of univariate 5-year renal allograft survival to compare those transplanted preemptively and those on dialysis for less than 6 months pretransplantation (both HD and PD were grouped together in a single dialysis group).

All statistical tests were two-sided and $\mathrm{p}$ value of $<0.05$ was considered to be statistically significant. Statistical analyses were performed in SPSS V.22.

\section{RESULTS}

\section{Patient characteristics}

A total of 2038 paediatric renal transplant recipients were analysed across the 16 years of this study with similar baseline characteristics (table 1). Six hundred seven (30\%) children were not on dialysis at the time of transplant (pre-emptive group), 642 (31\%) were on HD at the time of transplant and 789 (39\%) were on PD at the time of transplant.

\section{Overall patient and renal allograft survival analysis}

There was similar 1-year and 5-year patient survival across the three groups, but 5 -year renal allograft survival was significantly better in the pre-emptive group, compared with those on HD or $\mathrm{PD}$ at the time of transplant (figures 1 and 2).

The Cox proportional hazards regression model showed a significantly lower risk of 5 -year renal allograft failure in those not on dialysis at the time of transplant, compared with those on dialysis at the time of transplant (HD or PD), even after adjustment for donor type (HR 0.74 (95\% CI 0.55 to 0.99 ; $\mathrm{p}<0.05$ ). After adjustment for both donor type and recipient age, there is still a lower risk of 5-year renal allograft failure in those not on dialysis at the time of transplant, but this falls just short of statistical significance (HR 0.74 (95\% CI 0.55 to $1.002 ; \mathrm{p}=0.052$ ).

\section{Analysis of cumulative effect of time on dialysis on 5-year renal allograft survival}

Out of the 1431 children on HD or PD at the time of transplant, data on the length of time on dialysis pre-transplant were available for 991 of 1431 (69\%) children. We were unable to obtain data for the remainder of children in this registry study. More data on length of time on dialysis were available in the latter years of the study ( $80 \%$ data available for 2008-2015 cohort) compared with earlier years of the study $(56 \%$ data available for 2000-2007 cohort). Although the proportion of missing data differs across time, when the study cohorts are split the main finding (improved 5-year renal allograft survival for pre-emptive recipients) is maintained although without significance due to a loss of power. There was a negative correlation between length of time on dialysis pre-transplantation and absolute renal allograft survival with a longer period of time on dialysis associated with a lower renal allograft survival $(\mathrm{p}=0.002)$.

\section{Analysis of the effect of short period of time on dialysis on 5-year renal allograft survival}

There was no significant difference in 5-year renal allograft survival between those pre-emptively transplanted and 120 children on dialysis (HD or PD) for less than 6 months $(p=0.25$; figure 3 ).

\begin{tabular}{llllll}
\hline Table 1 & Baseline characteristics of 2038 paediatric renal transplant recipients included in this study & & \\
& $\begin{array}{l}\text { Pre-emptive } \\
(\mathbf{n = 6 0 7 )}\end{array}$ & $\begin{array}{l}\text { HD } \\
(\mathbf{n = 6 4 2 )}\end{array}$ & $\begin{array}{l}\mathbf{P D} \\
(\mathbf{n}=\mathbf{7 8 9})\end{array}$ & $\begin{array}{l}\text { Dialysis } \\
(\mathbf{n}=\mathbf{1 4 3 1})\end{array}$ & $\begin{array}{l}\text { Total } \\
(\mathbf{n}=\mathbf{2 0 3 8 )}\end{array}$ \\
\hline Recipient sex-male (\%) & $417(69)$ & $360(56)$ & $446(57)$ & $806(56)$ & $1223(60)$ \\
Mean recipient age (years) & 10.8 & 10.9 & 10.8 & 10.8 & 10.8 \\
\hline Living donors (\%) & $268(44)$ & $259(40)$ & $273(35)$ & $532(37)$ & $800(39)$ \\
Deceased donors (\%) & $339(56)$ & $383(60)$ & $516(65)$ & $899(63)$ & $1238(61)$ \\
\hline
\end{tabular}

$H D$, haemodialysis; $n$, number; $P D$, peritoneal dialysis. 


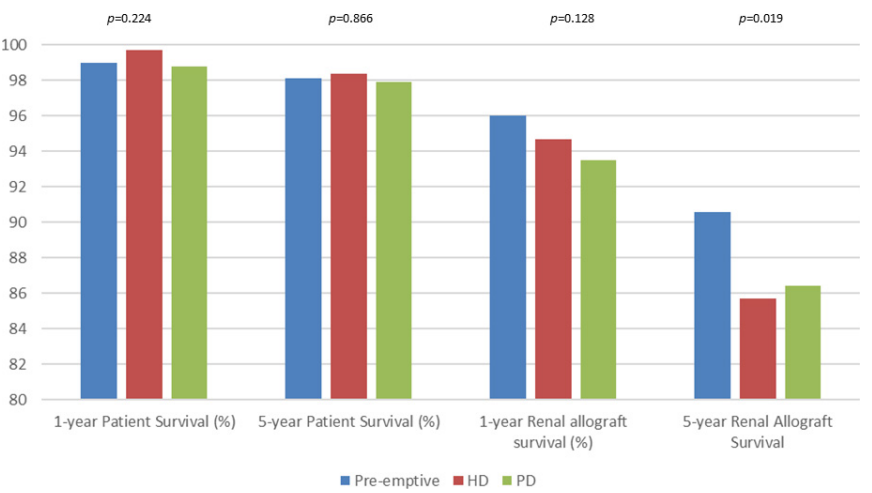

Figure 1 One-year and 5-year patient and renal allograft survival rates, split across those transplanted pre-emptively, those on $\mathrm{HD}$ and those on $\mathrm{PD}$ at the time of transplantation. $\mathrm{P}$ value corresponds to univariate log-rank test. HD, haemodialysis; PD, peritoneal dialysis.

\section{DISCUSSION}

This study has demonstrated that pre-emptively transplanted children have an improved 5-year renal allograft survival, compared with those on HD or PD at the time of transplant. In addition, we have shown that an increasing length of time on dialysis pre-transplantation is correlated with a lower renal allograft survival post-transplantation. However, our results do not show any significant difference between children transplanted pre-emptively and children on dialysis for less than 6 months pre-transplantation. The findings of our study support work done in the USA which similarly found an improved renal allograft survival in those pre-emptively transplanted; but in the study by Amaral et al, they found an increased risk of renal allograft failure even after short periods of time on dialysis pretransplant, while our study did not find this. ${ }^{11}$

One possible explanation for the primary finding of our study is that those children who are transplanted pre-emptively are an inherently different population at baseline. For example, they may show better compliance with medication, and therefore may be considered eligible for transplant sooner. Alternatively, those who are pre-emptively transplanted are likely to be those who have been followed up for a significant period of time by their paediatric nephrologist before they entered ESKD, therefore allowing their medical management to be optimised. Those

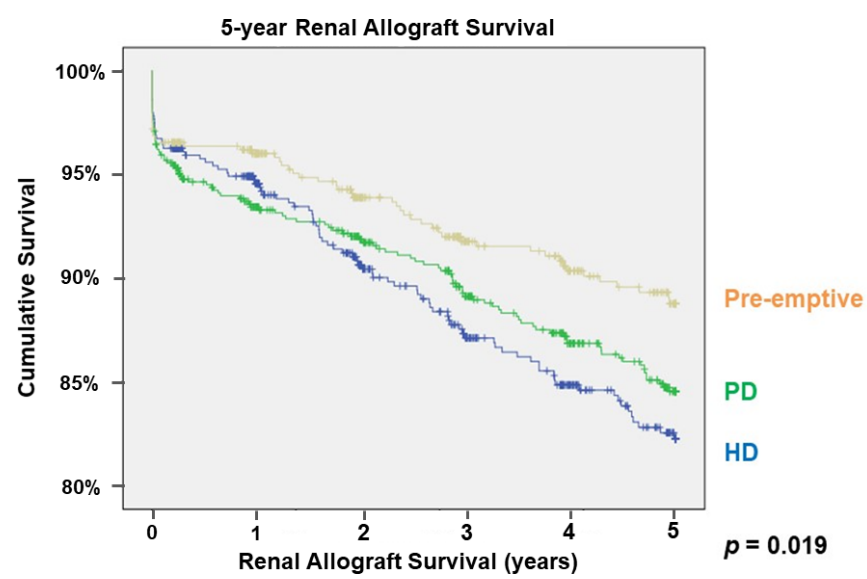

Figure 2 Kaplan-Meier curves showing 5-year renal allograft survival in those transplanted pre-emptively, those on $\mathrm{HD}$ at the time of transplant and those on PD at the time of transplantation. HD, haemodialysis; PD, peritoneal dialysis.

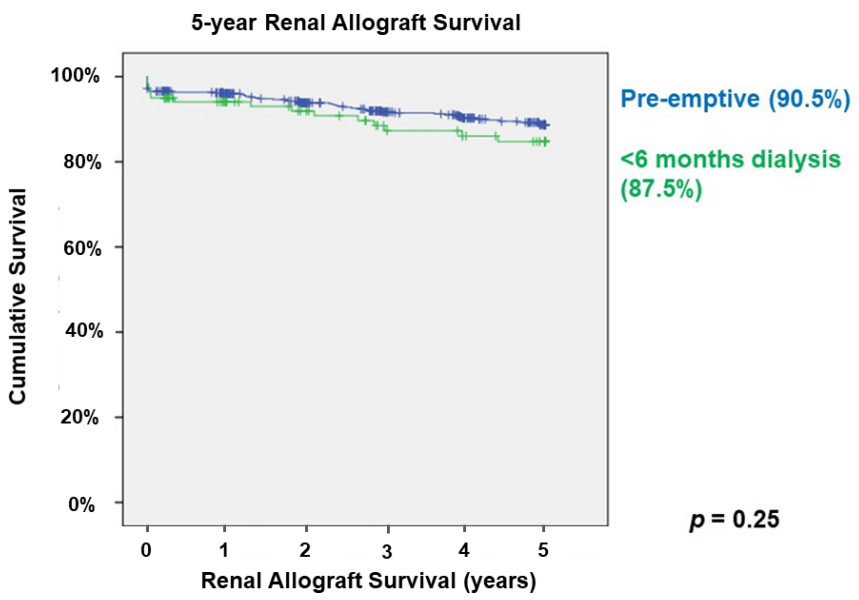

Figure 3 Kaplan-Meier curves showing 5-year renal allograft survival in those transplanted pre-emptively compared with 120 children on dialysis (HD or PD) for less than 6 months pre-transplantation. HD, haemodialysis; PD, peritoneal dialysis.

who required dialysis pre-transplantation may have a higher proportion of those patients who presented in ESKD, with the increased morbidity that can be associated with this. While these are possible theories, the registry nature of our study unfortunately does not let us explore these unmeasured potential baseline differences in detail.

In view of the above, we set out to find other pieces of evidence to support our primary finding being a true phenomenon. First, we found that an increasing length of time on dialysis pre-transplantation was correlated with a lower renal allograft survival suggesting there is a dose-response relationship and adds weight to the theory that some aspects of dialysis pre-transplantation have a causative effect in reducing renal allograft survival. Second, we analysed whether a short period of time on dialysis pre-transplant reduced renal allograft survival and we found that it did not. If the difference we observed in our primary analysis were due to unmeasured baseline differences, we would expect there to be a difference in renal allograft survival between those on dialysis for a short time as well, but this was not demonstrated. Therefore, we believe that the above provides us with further evidence supporting our primary finding, that some aspects of dialysis pre-transplantation have a negative effect on renal allograft survival.

It is not yet clear what aspects of dialysis pre-transplantation may affect renal allograft survival. It is well recognised that dialysis can have negative effects on cardiovascular health ${ }^{9}$ and it may be that this contributes to reduced renal allograft survival post-transplantation. In addition, clearance of some molecules (eg, $\beta-2$ microglobulin) is variable on dialysis, and this has been shown to contribute to mortality in adults. ${ }^{12}$ These molecules may be exhibiting a negative effect on the transplanted kidney, although it would be expected that this effect would resolve once the transplant occurs and renal function normalises. Other authors have also found evidence of increased T-cell alloimmunity in those with an increased dialysis vintage pre-transplant, which may, in part, account for inferior renal allograft outcomes. ${ }^{13}$

Not all forms of dialysis are equal and this is particularly important in paediatrics. Not only are there differences in clinical outcomes between different modalities, there are many relevant patient factors to consider such as quality of life. Our study shows that those on PD have better 5-year renal allograft survival than those on HD. In addition, newer modalities such 
as home HD (HHD) can provide optimised clinical and patient outcomes. ${ }^{14}$ Our study was unable to differentiate between those on in-centre HD and HHD based on its retrospective registry nature, so we are unable to draw conclusions on this subgroup, but it is likely that the effect size of our findings may diminish with improved dialysis care.

Our results are further limited by the retrospective registry data methods, with dialysis modality data only at the time of transplantation. For this reason, we have not focused on the differences between HD and PD in our analysis, as we do not have accurate data on change of dialysis modality over time.

In conclusion, our results show that children who receive a renal transplant pre-emptively have improved 5-year renal allograft survival, compared with those on dialysis at the time of transplantation. While dialysis is clearly a life-saving intervention that is essential in many cases, and short periods of time on dialysis do not appear to negatively affect renal allograft survival, our results support an even more enthusiastic drive to achieve pre-emptive renal transplant for children wherever possible.

Acknowledgements This project was supported by the National Institute for Health Research (NIHR) Biomedical Research Centers based at Great Ormond Street Hospital for Children NHS Foundation Trust and University College London. We are grateful to the NHS Blood and Transplant Kidney Advisory Group Paediatric Subgroup members and for the 13 paediatric nephrology units in the UK submitting data returns to the UK Transplant Registry.

Contributors SM conceived the idea for this study. All authors participated in research design. Data were collected from the UK transplant registry by KM, and analysed by KM and MM. The manuscript was drafted by MM and reviewed by all authors.

Funding The authors have not declared a specific grant for this research from any funding agency in the public, commercial or not-for-profit sectors.

Disclaimer The views expressed are those of the authors and not necessarily those of the NHS, the NIHR or the Department of Health.

Competing interests None declared.

Patient consent for publication Not required.

Ethics approval Data were fully anonymised and ethical principles were adhered to throughout the study. Data were transferred and handled according to the NHS Blood and Transplant policy, therefore, a separate ethical review was not required.

Provenance and peer review Not commissioned; externally peer reviewed.

Data availability statement Data are available upon reasonable request.
Open access This is an open access article distributed in accordance with the Creative Commons Attribution Non Commercial (CC BY-NC 4.0) license, which permits others to distribute, remix, adapt, build upon this work non-commercially, and license their derivative works on different terms, provided the original work is properly cited, appropriate credit is given, any changes made indicated, and the use is non-commercial. See: http://creativecommons.org/licenses/by-nc/4.0/.

\section{ORCID iDs}

Matko Marlais http://orcid.org/0000-0001-7503-7893

Stephen D Marks http://orcid.org/0000-0001-9850-8352

\section{REFERENCES}

1 Haller MC, Kammer M, Oberbauer R. Dialysis vintage and outcomes in renal transplantation. Nephrol Dial Transplant 2019;34:555-60.

2 Chesnaye NC, van Stralen KJ, Bonthuis M, et al. The association of donor and recipient age with graft survival in paediatric renal transplant recipients in a European Society for paediatric Nephrology/European renal Association-European dialysis and transplantation association registry study. Nephrol Dial Transplant 2017;32:1949-56.

3 Johnson RJ, Bradbury LL, Martin K, et al. Organ donation and transplantation in the UK-the last decade: a report from the UK national transplant registry. Transplantation 2014;97 Suppl 1:1-27.

4 Kim JJ, Marks SD. Long-Term outcomes of children after solid organ transplantation. Clinics 2014;69 Suppl 1:28-38.

5 Haller MC, Kainz A, Baer H, et al. Dialysis vintage and outcomes after kidney transplantation: a retrospective cohort study. Clin J Am Soc Nephrol 2017;12:122-30

6 Che X, Yang X, Yan J, et al. Effects of pretransplant peritoneal vs hemodialysis modality on outcome of first kidney transplantation from donors after cardiac death. BMC Nephrol 2018;19:235.

7 Kramer A, Jager KJ, Fogarty DG, et al. Association between pre-transplant dialysis modality and patient and graft survival after kidney transplantation. Nephrol Dial Transplant 2012:27:4473-80.

8 Prezelin-Reydit M, Combe C, Harambat J, et al. Prolonged dialysis duration is associated with graft failure and mortality after kidney transplantation: results from the French transplant database. Nephrol Dial Transplant 2019;34:538-45.

9 Rees L, Schaefer F, Schmitt CP, et al. Chronic dialysis in children and adolescents: challenges and outcomes. Lancet Child Adolesc Health 2017;1:68-77.

10 Meier-Kriesche $\mathrm{H}-\mathrm{U}$, Kaplan B. Waiting time on dialysis as the strongest modifiable risk factor for renal transplant outcomes: a paired donor kidney analysis. Transplantation 2002;74:1377-81.

11 Amaral S, Sayed BA, Kutner N, et al. Preemptive kidney transplantation is associated with survival benefits among pediatric patients with end-stage renal disease. Kidney Int 2016:90:1100-8.

12 Cheung AK, Rocco MV, Yan G, et al. Serum beta-2 microglobulin levels predict mortality in dialysis patients: results of the Hem0 study. J Am Soc Nephrol 2006;17:546-55

13 Augustine JJ, Poggio ED, Clemente M, et al. Hemodialysis vintage, black ethnicity, and pretransplantation antidonor cellular immunity in kidney transplant recipients. J Am Soc Nephrol 2007;18:1602-6.

14 Hothi DK, Stronach L, Harvey E. Home haemodialysis. Pediatr Nephrol 2013;28:721-30 